

ISSN: $2395-1958$

IJOS 2019; 5(1): 183-185

(C) 2019 IJOS

www.orthopaper.com

Received: 13-11-2018

Accepted: 15-12-2018

\section{Madamanchi Harsha}

Junior Resident, Department of Orthopaedics, Sri Devaraj Urs

Medical College, Tamaka, Kolar, Karnataka, India

\section{Eswara Reddy G}

Assistant Professor, Department of Orthopaedics, Sri Devaraj Urs Medical College, Tamaka, Kolar, Karnataka, India

\section{Arun HS}

Professor and Head of the Department, Department of Orthopaedics, Sri Devaraj Urs Medical College, Tamaka, Kolar, Karnataka, India

\section{Correspondence}

Eswara Reddy G

Assistant Professor, Department of Orthopaedics, Sri Devaraj Urs Medical College, Tamaka, Kolar, Karnataka, India

\section{Is intercrestal line and level of spine are same clinically and radiologically}

\author{
Dr. Madamanchi Harsha, Dr. Eswara Reddy G and Dr. Arun HS
}

DOI: https://doi.org/10.22271/ortho.2019.v5.i1d.34

\section{Abstract}

Background: The line joining the iliac crest superiorly and posteriorly is known as Intercrestal line. Various studies described that it crosses in the midline at L4-L5 intervertebral disc space level. Relationship of spinal level on palpation and imaging the iliac crest intercrestal line is not the same in particular the L4-L5 interspace.

Purpose: The purpose of this study is to compare the spinal level identified through palpation and imaging the iliac crest intercrestal line in adults.

Materials and Methods: Patients included low back pain undergoing epidural steroid injection at RL Jalappa Hospital and Research Center, between October 2018 to January 2019. Iliac crest posteriosuperiorly was palpated by the authors and the intercrestal line was drawn, then followed by imaging, the level of the spine in the midline identified and were recorded and compared.

Results: Our study shows that on palpation the L3-4 spinal level in 27 patients (48.2\%) and L4 spinal level in 22 patients $(39.2 \%)$ whereas on imaging the L4 spinal level in 33 patients (58.9\%) and L4-5 spinal levels in 21 patients $(37.5 \%)$, the intercrestal line formed through palpation tended to identify higher levels. On both methods the same level observed in $10.7 \%$ (6 patients) only.

Conclusion: Palpation and imaging method of iliac crest intercrestal line are the two methods to identify the spinal level. Clinically, the palpation of the intercrestal line to identify the L3-4 and L4 spinal levels rather than the L4 or L4-5 levels, particularly in patients with high BMI (body mass indices).

Keywords: Iliac crest, intercrestal line, palapation, imaging, spinal level

\section{Introduction}

For giving epidural injections in orthopaedics, anesthesiology and neurosurgery departments the intervertebral level identification is very important. To know the level of spine most commonly used bony landmark is iliac crest. The intercrestal line is the line joining posteriorly at the superior aspect of the iliac crests ${ }^{[1]}$. Various studies stated that the intercrestal line passes through the midline at L4-L5 intervertebral level ${ }^{[2-6]}$. Broadbent et al. described by iliac crest palpation method, the identification of correct spinal level is $29 \%$ only ${ }^{[6]}$. Jung et al. introduced another bony landmark as $10^{\text {th }}$ rib line in combination with iliac crest line ${ }^{[7]}$. However clinically identifying the exact intervertebral level is usually inaccurate by using iliac crest line ${ }^{[8,9]}$. The objective of the study is to compare the spinal level by using palpation method of iliac crest and C-Arm fluoroscopy imaging.

\section{Methods}

An observational prospective study was conducted from October 2018 to January 2019 at the RL Jalappa Hospital, Tamaka, Kolar, Karnataka, India. Written informed consent obtained from each patient and then enrolled in the study based on inclusion and exclusion criteria. Institutional ethical committee clearance obtained. Patients included in our study were with age more than $15 y$ rs who were having lower back pain undergoing epidural steroid injection under fluoroscopy guidance. Excluded from the study were patients with scoliosis, spondylolisthesis and any previous history of trauma to the spine where the anatomy of spine was compromised potentially.

Basic anthropometric variables age, sex, height, weight and body mass index (BMI) collected. Authors who are experienced, examined the patient by palpating the iliac crest on the 
Fluoroscopic table in prone position separately before imaging. A mark was made on the skin at postero-superior aspect of iliac crest and re-examined by each examiner and if there is any discrepancy of opinion, again remark the iliac crest intercresstal line was done as shown in figure. By using a radiopaque metal rod between the two marks for forming the intercrestal line followed by fluoroscopic images to check the spinal level at the midline and it was recorded. According to null hypothesis there is no difference between palpation and imaging of iliac crest intercrestal line with spinal level. We referred the level of spine as spinous process L4 or interspinous space L4-L5.

By using SPSS version 17.0 statistical analysis calculated. Descriptive variables calculated using mean and standard deviation. We correlated between basic anthropometric variables (age, height, weight, and BMI) and also the intercrestal line assessment. Statistical significance was considered at $P \leq 0.05$.



Fig 1: The iliac crest intercrestal line with markings at spinal level on prone position

\section{Results}

Fifty-six patients were included in our study with basic anthropometric variables (mean) are age 22-60 years (41 year), sex 26 males and 30 females, weight 53-100 kg (76.2 $\mathrm{kg}$ ), height $148-189 \mathrm{~cm}(168.2 \mathrm{~cm})$, body mass index $18-36$ $\mathrm{kg} / \mathrm{m}^{2}\left(24.6 \mathrm{~kg} / \mathrm{m}^{2}\right)$ which shown in Table 1 and Table 2

Table 1: Socio demographic Characteristics of study population

\begin{tabular}{|c|c|c|}
\hline Age & Number of patients & Percentage \\
\hline $22-35 \mathrm{yrs}$ & 18 & $32.1 \%$ \\
\hline $36-45 \mathrm{yrs}$ & 26 & $46.4 \%$ \\
\hline $46-60 \mathrm{yrs}$ & 12 & $21.4 \%$ \\
\hline
\end{tabular}

The following table demonstrates the distribution of population in different age groups and gender.

Table 2: Socio demographic Characteristics of study population

\begin{tabular}{|c|c|c|}
\hline Gender & Number of patients & Percentage \\
\hline Male & 26 & $46.4 \%$ \\
\hline Female & 30 & $53.7 \%$ \\
\hline
\end{tabular}

On palpation, the intercrestal line crosses the midline $8.9 \%$ at L3 spinous process, $48.2 \%$ at L3-L4 interspinous space, $37.5 \%$ at L4 spinous process, $3.5 \%$ at L4-L5 and no case at L5 spinous process.

Comparing the palpated level and the imaged level, palpated level is higher than the imaged level. One level higher in $71.4 \%$, two level in $17.8 \%$, same level in $10.7 \%$ shown in table 3 . The same level observed in 6 patients only shown in table 4.
Table 3: Shows the spinal levels on palpation of iliac crest intercrestal line by sex ( $\mathrm{SP}=$ spinous process, IS = inter-spinous space)

\begin{tabular}{|c|c|c|c|c|}
\hline Level & \multicolumn{2}{|c|}{ Palpation } & \multicolumn{2}{c|}{ Imaging } \\
\hline & Male & Female & Male & Female \\
\hline L3 SP & 0 & 5 & 0 & 0 \\
\hline L3-4 IS & 11 & 16 & 0 & 0 \\
\hline L4 SP & 13 & 9 & 16 & 17 \\
\hline L4-5 IS & 2 & 0 & 9 & 12 \\
\hline L5 SP & 0 & 0 & 1 & 1 \\
\hline TOTAL & 26 & 30 & 26 & 30 \\
\hline
\end{tabular}

Table 4: Shows the spinal levels identified by palpation and imaging of the intercristal line ( $\mathrm{SP}=$ spinous process, $\mathrm{IS}=$ interspinous space)

\begin{tabular}{|c|c|c|c|c|c|c|}
\hline Palpation & \multicolumn{7}{|c|}{ Imaging } \\
\hline & L3 SP & L3-4 IS & L4 SP & L4-5 IS & L5 SP & TOTAL \\
\hline L3 SP & 0 & 0 & 5 & 0 & 0 & 5 \\
\hline L3-4 IS & 0 & 0 & 23 & 4 & 0 & 27 \\
\hline L4 SP & 0 & 0 & 5 & 16 & 1 & 22 \\
\hline L4-5 IS & 0 & 0 & 0 & 1 & 1 & 2 \\
\hline L5 SP & 0 & 0 & 0 & 0 & 0 & 0 \\
\hline TOTAL & & & 33 & 21 & 2 & 56 \\
\hline
\end{tabular}

On no occasions did the palpated line identify a lower level than the imaging.

The palpated ICL identified the higher spinal levels in patients with higher BMI, the difference between the median values reach statistically significance $(p=0.03)$ shown in table 5. With the imaged ICL there was no significant difference between the spinal level and BMI.

Table 5: Correlation coefficient between the levels of intersection and patients' characteristics

\begin{tabular}{|c|c|c|c|}
\hline Variable & $\begin{array}{c}\text { Correlation } \\
\text { coefficient }\end{array}$ & $\begin{array}{c}\text { 95\% Confidence } \\
\text { Interval }\end{array}$ & $\begin{array}{c}\text { P } \\
\text { value }\end{array}$ \\
\hline Weight $(\mathrm{kg})$ & 0.19 & $0.13-0.46$ & 0.23 \\
\hline Height $(\mathrm{cm})$ & 0.24 & $0.46-0.09$ & 0.12 \\
\hline Body mass index $(\mathrm{kg} / \mathrm{m} 2)$ & 0.35 & $0.02-0.54$ & 0.03 \\
\hline
\end{tabular}

\section{Discussion}

For orthopaedics, anesthesiology and neurosurgery the exact spinal level identification through examination is very important. Several methods of identifying the exact spinal level by examination have been described. Ievins et al. included dropping a vertical line from the iliac crest uppermost part with side-lying position of the patient. Borley et al. identified the spinal level by construction of a line between the two PSISs. Jung et al. drawn a line between the lowermost margins of the tenth ribs.

In the 19th century, Jacoby and Tuffier first described the intercrestal line through palpation of the iliac crests, has proven to be the most popular method, with several authoritative textbooks referring to it as crossing the L4 or L4-5 spinal level ${ }^{[5]}$. By using X-rays and MRI scans of the lumbar spine many authors have attempted by using the intercrestal line to identify the exact spinal level. Our study found that the imaged ICL crossed the L4 spinous process in $58.9 \%$ and at the L4-5 interspinous space in $37.5 \%$ of adult patients with normal spinal anatomy and crossed the L5 spinous process in $3.5 \%$. Several authors demonstrated that through the palpation of bony landmarks it is difficulty to identifying the exact spinal level. Agreement between clinicians on the estimated level through palpation, however, has been found to be good, at 60\% (Broadbent et al. 2000), and thus it appears that it is the use of L4 or L4-5 as the guide 
rather than the execution of the method of forming the line that appears to lead to errors. Only Ievins et al. in 1991 has found a reasonable percentage $(78.3 \%)$ identification of the correct spinal level compared with half-Tuffier's line $(59.3 \%)$ but the study sample was small and cadavers, rather than live subjects, were used.

Factors that have been shown to influence poor identification of the correct spinal level include selection of a high lumbar spinal level and obesity. Positioning of the patient in a sitting or lateral position does not affect the accuracy even though in nearly half the patients, the spinal level intersected by the ICL on imaging moves downward by one level on full lumbar flexion.

The present study may help to explain the frequent inaccurate identification of the correct spinal levels found in previous studies. The palpated ICL identifies the L3-4 intraspinous space in the majority of cases $(48.2 \%)$. The imaged ICL identifies majorly at the L4 and L4-5 spinal levels (96.4\%). We found that the difference between the palpation and imaging iliac crest intercrestal line were not significant with L4 spinal level only. Whenever identifying the spinal level, it is important to distinguish between these two methods of identifying the intercrestal line. The subcutaneous tissue between the bony landmarks and the palpating hand is the main difference. According to Bartali et al. and Gallagher et al. females who have more percentage body fat with high BMI were found to have proportionately higher levels identified on palpation. We included patients with normal lumbar spine anatomy but Ford \& Goodman and O'Driscoll et al. stated the prevalence rate of $8-15 \%$ expected for sacralization or lumbarization of the spine, Frederickson et al. stated the prevalence up to $6 \%$ for spondylolisthesis, lumbar scoliosis or loss of vertebral height. Limitations of the present study were the small sample size. A larger scale of study with various patients is still necessary.

\section{Conclusion}

Our study concluded that by using the iliac crest intercrestal line, on palpation, the most frequently identified spinal level is the L3-4 interspinous space, whereas, with imaging method the most frequently identified spinal level is the L4 spinous process and the L4-5 interspinous space. Both methods were shown the same spinal level in $10.7 \%$ only. Using palpation and imaging to construct the intercrestal line considered as two methods to identify the exact spinal level. In patients with high BMI the method of palpating iliac crest intercrestal line is not appropriate to identify the exact spinal level. It is always better to use image method of iliac crest intercrestal line to identify the exact spinal level in patients with high body mass index BMI.

\section{Declaration of patient consent}

We obtained the consent from the patients who are all included in this study. They understand that their names and initials will not disclose.

\section{Financial support and sponsorship}

Nil

\section{Conflicts of interest}

There are no conflicts of interest

\section{References}

1. Kim JT, Jung CW, Lee JR, Min SW, Bahk JH. Influence of lumbar flexion on the position of the intercrestal line.
Reg Anesth Pain Med. 2003; 28:509-511.

2. Lirk P, Hogan Q. Spinal and epidural anatomy. In: Wong CA (Ed). Spinal and Epidural Anesthesia, 1st ed. Chicago: McGraw-Hill Companies Inc, 2007, 1-27.

3. Cook TM, Counsell D, Wildsmith JA. Royal College of Anaesthetists Third National Audit Project. Major complications of central neuraxial block: report on the Third National Audit Project of the Royal College of Anaesthetists. Br J Anaesth. 2009; 102:179-90.

4. Snider KT, Kribs JW, Snider EJ, Degenhardt BF, Bukowski A, Johnson JC. Reliability of Tuffier's line as an anatomic landmark. Spine. 2008; 33:161-5.

5. Chakraverty R, Pynsent P, Isaacs K. Which spinal levels are identified by palpation of the iliac crests and the posterior superior iliac spines? J Anat 2007; 210:232-6.

6. Broadbent CR, Maxwell WB, Ferrie R, Wilson DJ, Gawne-Cain M, Russell R. Ability of anaesthetists to identify a marked lumbar interspace. Anaesthesia. 2000; 55:1122-6.

7. Jung CW, Bahk JH, Lee JH et al. The tenth rib line as a new landmark of the lumbar vertebral level during spinal block. Anaesthesia. 2004; 59:359-63.

8. Furness G, Reilly MP, Kuchi S. An evaluation of ultrasound imaging for identification of lumbar intervertebral level. J Anaesthesia. 2002; 57:277-280 